\title{
Aberration-free measurements of the visibility of isoluminant gratings
}

\author{
Nobutoshi Sekiguchi, ${ }^{*}$ David R. Williams, and David H. Brainard ${ }^{\dagger}$ \\ Center for Visual Science, University of Rochester, Rochester, New York 14627
}

Received August 19, 1992; revised manuscript received April 7, 1993; accepted April 8, 1993

\begin{abstract}
We developed a new apparatus and psychophysical technique to extend isoluminant contrast-sensitivity measurements to high spatial frequencies. The apparatus consists of two identical laser interferometers that are designed to produce phase-locked two-color interference fringes on the retina without the influence of diffraction and most aberrations in the eye. However, even with interferometry, transverse chromatic aberration of the eye can produce a wavelength-dependent phase shift in the interference fringes, which can be exaggerated by head movements. To reduce the effect of head movements, isoluminant red and green interference fringes of equal spatial frequency and orientation were drifted slowly in opposite directions to guarantee a purely isochromatic (in phase) and a purely isoluminant (out of phase) stimulus during each cycle of stimulus presentation. With this technique we found that observers could resolve red and green stripes at spatial frequencies higher than 20 cycles per degree (c/deg) (20-27 c/deg), substantially higher than has previously been reported. This places a lower bound on the sampling density of neurons that mediate color vision. At all spatial frequencies, even those above the isoluminant resolution limit, a relative phase of the red and the green components could be found that obliterated the appearance of luminance modulation at the fringe frequency. Above the resolution limit, red-green-isoluminant interference fringes are seen as spatial noise, which may be chromatic aliasing caused by spatial sampling at some stage in the chromatic pathway.
\end{abstract}

\section{INTRODUCTION}

The basic properties of the visual system for detecting spatial modulation in chromaticity can be described by contrast-sensitivity functions for isoluminant color directions. Many investigators have reported contrastsensitivity measurements for isoluminant gratings. ${ }^{1-9}$ In these previous measurements, however, the range of spatial frequencies over which isoluminant contrast sensitivity could be measured was restricted by several factors. One restriction comes from technical difficulties in producing high-contrast isoluminant gratings with CRT displays, which many studies have used. This is because spectrally broadband phosphors in CRT displays lower the effective contrast of isoluminant but not of isochromatic stimuli. In addition, CRT displays cannot produce high luminance levels, which restricts the ability to measure performance at higher spatial frequencies. Perhaps the most important restriction is the contrast reduction caused by optical blurring in the eye, particularly by axial and transverse chromatic aberration.

Mullen ${ }^{7,8}$ and Anderson et al. ${ }^{9}$ separated the positions of the different wavelength components of their stimulus to compensate for both axial and transverse chromatic aberrations. Even with this compensation, their measurements did not extend beyond 10 cycles per degree (c/deg).

In this paper we show that these spatial-frequency restrictions can be overcome by producing an isoluminant stimulus with laser interferometry and by measuring performance with a new psychophysical technique. The advantages of using laser interferometry are as follows:

1. Interferometry permits contrast-sensitivity measurements at high spatial frequencies, since it can avoid the influence of optical blurring. ${ }^{10-12}$ Moreover, particularly for isoluminant conditions, this technique can avoid luminance artifacts produced by axial chromatic aberration.

2. Laser sources emit monochromatic light, which permits higher chromatic contrasts than can easily be achieved with CRT displays.

3. A laser source can easily produce the high luminances at which contrast sensitivity is maximal.

Although the use of interferometry solves many of the technical problems associated with the production of highcontrast isoluminant gratings, it does not eliminate the possibility of a luminance artifact caused by a phase error between the two grating components. Independently of how isoluminant gratings are produced, this sort of phase error can be introduced by transverse chromatic aberration of the eye. The effect can be exaggerated by head movements. ${ }^{13}$ In order to reduce the influence of the phase artifacts, we have developed a new psychophysical technique to guarantee the presentation of a pure isoluminant stimulus despite the small head movements of an observer clenching a bite bar.

In experiment 1 we validate the new psychophysical technique. In experiment 2 we use our technique to measure the foveal resolution limit for isoluminant red-green fringes. We find that observers can resolve such fringes at spatial frequencies of $20-27 \mathrm{c} / \mathrm{deg}$, substantially higher than has previously been measured. In the companion paper ${ }^{14}$ we apply this technique to measure both isoluminant and isochromatic contrast-sensitivity functions and to compare the efficiency of the visual system for detecting these two types of stimulus. 


\section{APPARATUS: TWO-CHANNEL LASER INTERFEROMETER}

Williams ${ }^{12}$ improved the design of a laser interferometer for vision research to permit computer control of the parameters that specify the spatial and temporal properties of an interference fringe. Here we have made further improvements of the previous design, constructing two identical interferometers that produce two fringes on the retina with a relative phase that can be precisely controlled. Our new interferometers are designed to phase lock the two fringes and to minimize fringe distortion. To do this we capitalized on the benefits of common-path interferometry. ${ }^{15,16}$ For most of their optical paths, the two interfering beams traverse the same components, so that the resulting interference fringe is much less affected by outside disturbances than in an interferometer in which the two beams are widely separated. Furthermore, the fringe distortion produced by optical components is reduced in the common-path scheme because the two interfering beams share the same components.

Whereas the need for stability favors the common-path scheme, the control of all the fringe parameters, especially contrast and temporal frequency, requires the separation of the interfering beams into different optical paths in one portion of the interferometer. However, the two beams can still share a large portion of their optical paths if they are tagged with different polarization states. ${ }^{16}$ We incorporated this concept into our design.

\section{Optical Design}

The new apparatus consists of two similar interferometers, each of which has its own laser source. Typically, the laser source of each interferometer is of a different wavelength so that one can produce chromatic fringes by superimposing the interferometers' outputs. The spatial frequency, orientation, contrast, phase, and temporal frequency of the two interference fringes can be controlled independently in each interferometer. All these parameters are under computer control, except for spatial frequency and orientation for one of the interferometers, which are controlled manually. Otherwise the two devices are identical.

Figure 1 shows one of the two interferometers. A polarized laser beam, produced by the laser source, LS, first passes through a half-wave retardation plate, HP, and a linear polarizer, LP1. The orientation of LP1 determines the relative intensity of two orthogonally polarized components, $S$ and $P$, which in turn determine the maximum contrast of the interference fringe. Usually LP1 is adjusted to equate the two polarization components so that unity contrast is obtained. The rotation of HP about the optical axis rotates the plane of polarization. The polarization direction relative to the plane of preferred transmission of LP1 determines the total intensity of the interference fringe, preserving the relative intensity of the two polarized components. The laser beam is divided in two by the polarization beam splitter, PBS1. The $S$ component is reflected and the $P$ component is transmitted. Each beam passes through an acousto-optic modulator, AOM, that controls the contrast and the spatial phase of the interference fringe (see below). The two beams are recombined by the polarization beam splitter, PBS2, which preserves their polarization properties. The combined beams are expanded by a spatial filter, SPF, which also functions to remove the spatial noise from the beams, and are collimated by lens L1. (Other lenses are denoted L2-L6.)

The beam is divided again by the polarization beam splitter, PBS3, and the $S$ and $P$ components travel in the triangular common-path interferometer clockwise and counterclockwise, respectively, until they are recombined by PBS3. Inside the triangle, the two beams cross inside the glass cube, $\mathrm{C}$, traveling in nearly opposite directions. Rotations of the glass cube control fringe spatial frequency and orientation as described below. The beams are combined by PBS3 and then pass through a linear polarizer, LP2, the orientation of which is set to transmit an equal amount of the $S$ and the $P$ components and also to be perpendicular to the orientation of LP2' in the other interferometer. The beams from the two interferometers are combined by beam splitter BS1 and go through an analyzer, LP3. The analyzer permits control of the relative intensity of the two interference fringes that are orthogonally polarized.

To reduce the influence of the speckle noise, which inevitably exists in coherent light, one can add a spatially uniform incoherent background to the interference fringes. The incoherent background is produced by a typical twochannel Maxwellian optical system, not shown in the diagram. The incoherent light is produced by a tungsten light source and passes through monochrometers, one in each channel. It is combined with the interferometric beams by beam splitter BS2. The beams from the two interferometers and the incoherent channels are focused on the observer's pupil plane by a final (Maxwellian) lens, L6. The size of the incoherent image at the pupil plane is smaller than $2 \mathrm{~mm}$ so that no light is occluded by the

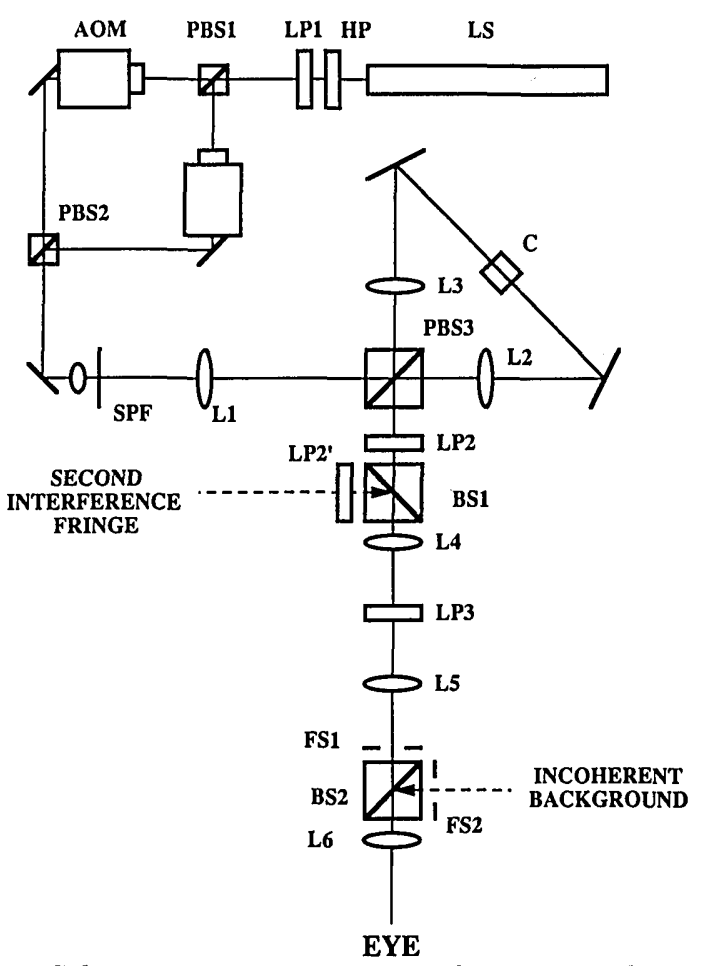

Fig. 1. Schematic diagram of one of the two polarization interferometers. 
iris. The field stops, FS1 and FS2, are conjugate with the retina.

We isolated the whole system from vibrations transmitted through the floor by floating the optical table on an array of 10-in.-(25-cm) diameter inner tubes. Furthermore, the two interferometers are close together [built on a $3 \mathrm{ft} \times 3 \mathrm{ft}(1.1 \mathrm{~m} \times 1.1 \mathrm{~m})$ optical breadboard anchored to the floating table] so that any variation in fringe position of one interferometer tends to occur in the other, with the relative phase relationship remaining constant. We surrounded most of the optical components by acrylic enclosures to reduce disturbances resulting from air circulation. This is particularly effective for the pathway between PBS1 and PBS2, which is only the place where the two interfering beams in each interferometer are separated.

\section{Spatial Frequency and Orientation}

The fringe spatial frequency, $f$ (c/deg), is proportional to the separation, $d(\mathrm{~mm})$, between the two point sources in the entrance pupil and is approximately represented by

$$
f=\frac{\pi d}{180 \lambda}
$$

where $\lambda(\mathrm{mm})$ is the wavelength of a laser source. Fringe orientation is perpendicular to the orientation of the two point sources in the entrance pupil. The glass cube, C, refracts the two beams, displacing them by equal amounts in opposite directions. The amount and the direction of this displacement depend on the orientation of the cube. The two rotational axes of the glass cube are perpendicular to each other and to the optical axis, permitting control of the positions of the point sources in two dimensions. The glass cube is conjugate with the entrance pupil of the eye, so that the beam displacements that it produces are faithfully rendered in the pupil. Since the displacements are always symmetric about the optical axis, spatial frequency and orientation can be controlled without the need for realigning the observer's eye. The spatial frequency and orientation of one of the fringes can be controlled by a computer, while those of the other fringe are controlled manually with a fine micrometer screw that provides continuous adjustment of spatial frequency and orientation. In the present system both a $\mathrm{He}-\mathrm{Ne}$ laser $(632.8 \mathrm{~nm})$ and a $\mathrm{He}-\mathrm{Cd}$ laser (441.6 $\mathrm{nm}$; used in the study reported in the companion paper ${ }^{14}$ ) are aligned as potential sources for the interferometer with the computer-controlled cube, and an $\mathrm{Ar}^{+}$laser (514.5 $\mathrm{nm}$ ) is the source for the interferometer with the manually controlled cube. We will refer to the former as interferometer $\mathrm{A}$ and the latter as interferometer $B$.

The fringe spatial frequency and orientation for interferometer A were calibrated by two different methods. In the first method, a Ronchi ruling of known spatial frequency was placed at field stop FS1 shown in Fig. 1. The observer saw the interference fringe superimposed upon the ruling and adjusted the fringe spatial frequency to minimize the frequency of the moiré pattern generated by them. We used five Ronchi rulings $(8.6,12.9,17.2$, 21.5 , and $25.8 \mathrm{c} / \mathrm{deg}$ ) to calibrate both horizontal and vertical fringes produced by a He-Ne laser $(632.8 \mathrm{~nm})$ and a $\mathrm{He}-\mathrm{Cd}$ laser $(441.6 \mathrm{~nm})$. In the second method, we calibrated the higher spatial frequencies by measuring the separation between the two interfering point sources at the pupil plane with a narrow slit attached to a micrometer translational stage. This was done for both vertical and horizontal interference fringes $(632.8 \mathrm{~nm})$ every $30 \mathrm{c} / \mathrm{deg}$ up to and including $150 \mathrm{c} / \mathrm{deg}$. The resulting frequency error was measurable at very high spatial frequencies but never exceeded $3 \%$.

\section{Contrast}

The fringe contrast is controlled by the pulse-overlap technique described by Williams. ${ }^{12}$ Each laser beam is chopped by an acousto-optic modulator (AOM) into 1-ms pulses at $500 \mathrm{~Hz}$. The contrast of the fringe is controlled by the temporal overlap of the two pulses, one from each AOM. That is, when the two pulses occur simultaneously, the fringe contrast is $100 \%$. Reducing the overlap in time of the pulse pair reduces the contrast, which reaches 0 contrast when the pulses do not overlap at all. This method permits control of the contrast without changing the total amount of light arriving at the retina.

The fringe contrast for both interferometers was calibrated with a high-resolution CCD camera. The interference fringe was imaged on the CCD array, and we processed the output from the central $512 \times 512$ pixels with a fast-Fourier-transform program to obtain its frequency spectrum. We then filtered the spectrum near $\pm 5 \%$ of the fundamental frequency, transformed the result back to the space domain, and calculated the Michelson contrast. Figure 2 shows the measured fringe contrast as a function of the nominal contrast defined by the ratio of pulse overlap. The results for the two interferometers are quite similar. The results indicate a residual contrast of $\sim 4 \%$ at low contrast levels. This residual contrast is generated by polarization cross talk between the two beams of the interferometer. We believe that most of this cross talk is produced by the microscope's objective lens in the spatial filter and by incomplete extinction by the polarization beam splitters. All the contrast-sensitivity measurements described below were made in a contrast range well above that for which the residual contrast was problematic. This was done with the use of an incoherent background. The background reduced the residual contrast below interference-fringe contrast threshold for the ob-

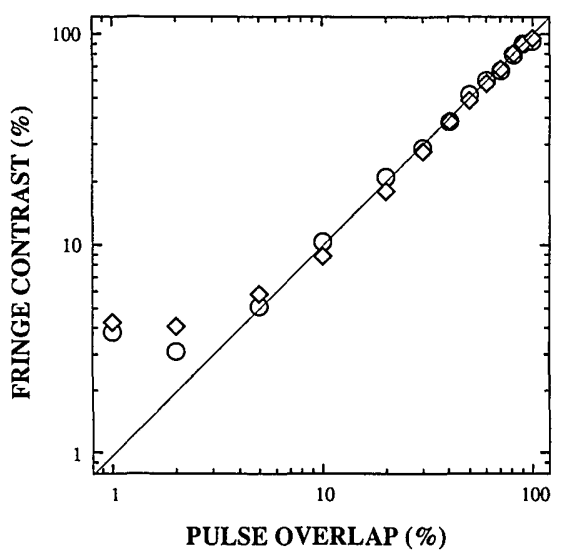

Fig. 2. Contrast of the interference fringes produced by the two polarization interferometers as a function of the temporal overlap between a pair of pulsed interfering beams. Circles and diamonds represent the results for interferometer $\mathrm{A}$ and interferometer B, respectively. 
server $(\sim 0.5 \%) .{ }^{12,17}$ At higher contrast levels the actual contrast is proportional to the nominal contrast, although it is very slightly lower (1-2\%). We attributed the lower fringe contrast to the presence of speckle noise.

\section{Phase (Drift Rate)}

The AOM's also controlled the spatial phase of the interference fringes as described by Coletta et $a .^{15}$ The relative phase shift in the $40-\mathrm{MHz}$ signals that drive the AOM's produces a delay in one of the wave fronts relative to the other to produce a spatial phase shift in the resulting interference fringe. To produce a drifting interference fringe, we update the phase shift every $2 \mathrm{~ms}(500 \mathrm{~Hz})$, which permits smooth drift at any temporal frequency within the visible range.

\section{Fringe Stability}

For a pure isoluminant interference fringe to be produced, the phases of the two component fringes must be stable. A phase shift of one fringe relative to the other causes a luminance artifact that affects measured isoluminant contrast sensitivity. When the temporal frequency was nominally zero, the fringe stability, in terms of the relative phase shift between the two interference fringes, was measured with the high-resolution CCD camera. A circular aperture placed at the position conjugate to the CCD array in each interferometer was aligned so that two fringes were adjacent to each other on the CCD array. 5 -c/deg fringes of unity contrast produced by a $\mathrm{He}-\mathrm{Ne}$ laser $(632.8 \mathrm{~nm})$ and $\mathrm{an} \mathrm{Ar}^{+}$laser $(514.5 \mathrm{~nm})$ were tested. We took an image containing both fringes, and we processed each fringe image independently with a fastFourier-transform program to obtain phase information. Images were collected every $10 \mathrm{~min}$ over a period of $2 \mathrm{~h}$. The results show that the relative phase shift is very slow, approximately $2 \mathrm{deg}$ of phase angle per minute. These slow phase shifts were probably caused by thermal drift in the optical system.

\section{Fringe Distortion}

The fringe distortion produced by the optical components in the apparatus was estimated as the minimum moiré frequency produced between the fringe and the retinal image of a Ronchi ruling placed at field stop FS1 shown in Fig. 1. For both interferometers the resulting moiré pattern was less than 1 cycle across the circular test field $6 \mathrm{deg}$ in diameter. The local phase error that was observed when two fringes with the same spatial frequency and orientation were added together on the retina was measured in terms of the frequency of a nonlinear distortion product. ${ }^{19-21}$ The measurement was done with 632.8and 514.5-nm interference fringes. We confirmed that, with a careful adjustment of the manually controlled glass cube, we could achieve no measureable local phase error within at least a central 2-deg field.

\section{Alignment Procedure}

The procedure for aligning the observer's eye in the apparatus was similar to that described by Williams. ${ }^{12}$ First, on the basis of the alignment procedure in the conventional Maxwellian view, the observer's pupil center was aligned to the apparatus. Second, some observers required a small adjustment along the system's optical axis to minimize the granular appearance of the test field. This minimization occurs when the point sources are focused on the corneal surface. Third, the observer's eye was realigned both horizontally and vertically with respect to its Stiles-Crawford maximum in the entrance pupil, instead of the pupil center, so that the effectiveness of the two beams from each interferometer could be kept equal. The circular field stop placed at FS1 was temporarily moved to a position that rendered it out of focus, and the fringe spatial frequency was set to $\sim 90 \mathrm{c} / \mathrm{deg}$. This procedure produces a double image of the field stop on the retina: horizontal and vertical fringes produce vertical and horizontal displacements, respectively. The horizontal or vertical positioning was achieved by making the two fields, displaced horizontally or vertically, equally bright.

\section{ARTIFACTS IN THE GENERATION OF TWO-COLOR GRATINGS}

One persistent problem in producing isoluminant gratings is the possibility of generating a luminance artifact that is due to chromatic aberration in the eye. When isoluminant gratings are produced by simply adding red and green gratings of the same spatial properties, a luminance artifact can be introduced in any of three ways: as a result of (1) an amplitude difference, (2) a magnification difference, or (3) a phase shift between the two components.

If one component is blurred more than the other, a luminance modulation appears in the sum. Such amplitude differences can occur with conventional gratings because of axial chromatic aberration and diffraction of the eye, which demodulate gratings by an amount that depends on their wavelength. Many investigators have used achromatizing lenses to correct axial chromatic aberration.,22-24 We used interference fringes, which not only avoid axial chromatic aberration but also avoid diffraction and monochromatic aberrations of the eye.

If there is a difference in spatial frequency between the two components, a luminance modulation also appears in the sum. Such a magnification (spatial-frequency) error can occur both with conventional gratings and with interference fringes because of transverse chromatic aberration of the eye, which displaces the principal rays of the gratings by an amount that depends on their wavelength and incident angle. Note that the achromatizing technique often used with conventional gratings to correct for axial chromatic aberration does not correct for transverse chromatic aberration. ${ }^{25,26}$ Therefore, whether one uses conventional gratings or interference fringes, one must take additional steps to equate the spatial frequency of the two component gratings. In our experiment each observer equated the spatial frequencies of the fringes from the two interferometers.

If the phase of one component is shifted relative to that of the other, a spurious luminance modulation also appears in the sum. Such phase shifts can occur both with conventional gratings and with interference fringes because of displacements of the light entering the pupil with respect to the achromatic axis of the eye's optics. In the Maxwellian view head and eye movements cause such displacements. In Appendix A we describe a ray-tracing estimate of how large the relative phase shift that is due to the observer's head and eye movements might be for 
(a)

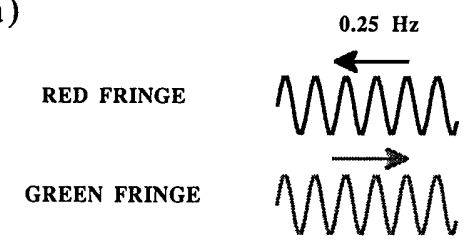

(b)

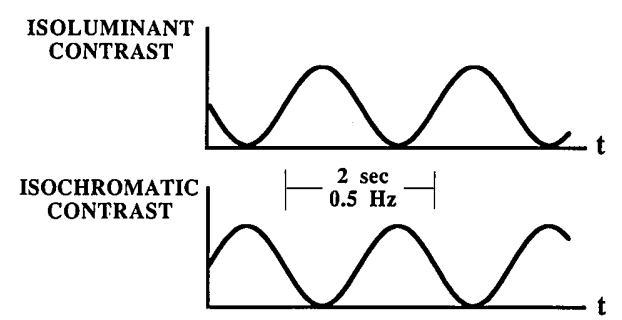

Fig. 3. Oppositely drifting red-green interference fringes. (a) Red and green fringes drifting in opposite directions at $0.25 \mathrm{~Hz}$, (b) resulting isoluminant and isochromatic contrast modulated sinusoidally at $0.5 \mathrm{~Hz}$.

red-green interference fringes. We show that head movements are expected to produce much larger effects than are eye movements and that these effects become particularly disruptive at higher spatial frequencies. We also provide estimates of the effects of head movements on measured isoluminant contrast sensitivity. Our computations suggest that head movements prevent measurement of isoluminant contrast sensitivity at high spatial frequencies with static interference fringes. This result is consistent with the observers' subjective observations: at spatial frequencies higher than 8-10 c/deg, most observers found it difficult to preserve the relative phase of the red and the green interference fringes. At low spatial frequencies they had no difficulty.

\section{PSYCHOPHYSICAL TECHNIQUE}

\section{Drifting Technique}

In order to reduce the effect of head movements, we have developed a new psychophysical technique, which we refer to simply as the drifting technique. In this technique a red (632.8-nm) and a green $(514.5-\mathrm{nm})$ interference fringe of equal spatial frequency, orientation, and space-averaged retinal illuminance were drifted slowly in opposite directions at $0.25 \mathrm{~Hz}$, as shown in Fig. 3(a). When the fringes are in phase, the stimulus consists only of luminance variations in the yellow (mixture of red and green) field. When they are out of phase, the stimulus consists of alternating red and green bars. Each condition appears once during each temporal cycle, as shown in Fig. 3(b). This stimulus can be thought of as the sum of an isochromatic and an isoluminant grating, with the contrast of each modulated sinusoidally at $0.5 \mathrm{~Hz}$.

The advantage of this oppositely drifting fringe stimulus over conventional static grating stimuli is that we can always guarantee a purely isochromatic and a purely isoluminant stimulus during each cycle of stimulus presentation. Eye or head movements can slightly advance or delay when these events occur, but they do not eliminate them. This technique avoids the uncertainty in adjusting the relative phase of the two interference fringes that exists when static fringe stimuli are used, particularly at high spatial frequencies and when they are presented in the parafovea. The phase uncertainty may cause a luminance artifact when we try to add the two interference fringes 180 deg out of phase, or it may reduce the luminance contrast when we try to add them in phase. These factors could distort the estimates of both isoluminant and isochromatic contrast sensitivities.

In all cases the contrasts of the two fringes were equal, and the observer adjusted the contrasts of the two fringes simultaneously to find threshold. In order to measure isochromatic contrast sensitivity, the observer adjusted the contrast until the luminance modulation was just detectable. In order to measure isoluminant contrast sensitivity, the observer adjusted the contrast until the red and green modulation was just detectable. These two tasks turned out to be easy for observers to perform.

The criterion for determining the isoluminant contrast threshold is based on the appearance of chromatic stripes. This itself may help to avoid the influence of a luminance artifact, but we still wish to deliver the highest-possible isoluminant (chromatic) contrast to the observer, which occurs only when the red and green fringes are exactly out of phase. The underlying assumption in this technique is that the observer can distinguish chromatic events from luminance events and independently determine threshold for each in a single stimulus. This assumption is tested below in experiment 1 .

\section{Flicker Photometry}

The space-averaged retinal illuminances of the two interference fringes were equated for each observer by flicker photometry. The red (632.8-nm) and the green (514.5$\mathrm{nm}$ ) interference fringes were set at zero contrast and zero spatial frequency and were presented alternately at $12 \mathrm{~Hz}$. The observer fixated the center of the field and adjusted an analyzer, LP3 in Fig. 1, to control the relative intensity of the red and the green lights to minimize flicker. The total retinal illuminance was kept constant at 500 trolands (Td). The test stimuli were presented in a sharp-edged circular field, and no background field was used. In Appendix B we estimate the distortion in measured isoluminant contrast sensitivity caused by errors in flicker photometry. The effects are less severe than those caused by the relative phase shift between the two fringe components. We estimate that variability in flicker settings, which may appear depending on the spatial frequency or stimulus size, would not affect measured isoluminant contrast sensitivity by more than 0.1 log unit.

\section{Test Stimulus and Procedure}

As shown in Fig. 4, we presented all fringes in a circular field windowed by a Gaussian aperture to eliminate the possibility of an edge artifact. ${ }^{27,28}$ The Gaussian luminance profile was produced by placement of a photographic transparency at field stop FS1 shown in Fig. 1. The twodimensional Gaussian density distribution was generated by a computer, and the image was directly photographed by a film recorder. The size of each pixel is $0.48 \mathrm{arcmin}$, which is not resolved by the eye's optics. Since the typical photographic process is known to be nonlinear, we calibrated the Gaussian filters by measuring with a highresolution $\mathrm{CCD}$ camera the intensity profile of the light 


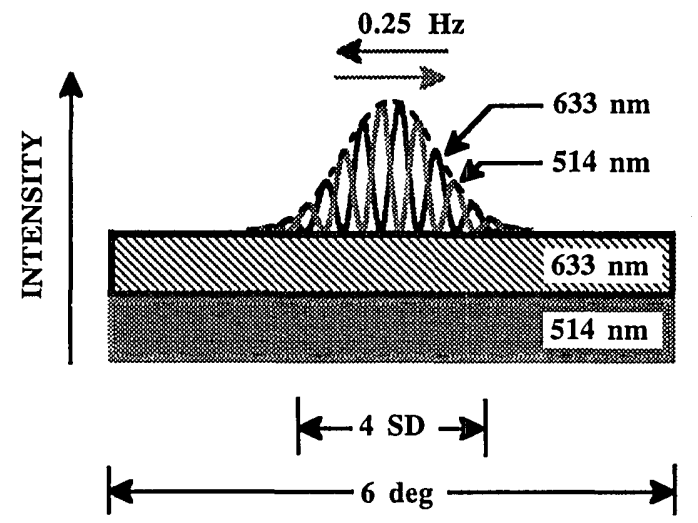

Fig. 4. Luminance profile of the red-green stimulus.

transmitted through the filter. The resulting density profile for each filter was well approximated by a Gaussian function. Five cycles of the grating stimulus were presented within 4 standard deviations of the Gaussian function for all spatial frequencies.

The test fringes were superimposed upon a spatially uniform incoherent background 6 deg in diameter, which consisted of the same wavelength pair as the fringes. The retinal illuminances of the two background components were equal. The purpose of the incoherent background was to reduce the influence of speckle masking. ${ }^{17}$ In the measurements the coherent fraction, the proportion of the total retinal illuminance that is coherent light, was $9 \%$. The total retinal illuminance was $500 \mathrm{Td}$ at the center of the Gaussian envelope.

For a given spatial frequency the observer adjusted the glass cube in interferometer $B$ to match the spatial frequency and orientation of the two interference fringes. Then a Gaussian filter was placed at field stop FS1 shown in Fig. 1, and a background field was presented to the observer. The contrast of both fringes was set at 0 , and the observer adapted to the test field for $1 \mathrm{~min}$.

\section{EXPERIMENT 1}

We examined whether the new psychophysical technique provides estimates of contrast sensitivity for both isoluminant and isochromatic stimuli that agree with those provided by conventional techniques. The measurements were limited to relatively low spatial frequencies, at which the effects of the observer's head movement are not significant (see details in Appendix A).

\section{Method}

Contrast sensitivity for isoluminant and isochromatic interference fringes was measured with three different methods. The first method was the drifting technique described above. In the second and third methods the test stimulus was the sum of static red and green fringes, instead of drifting fringes. In the second method the contrast sensitivities were measured with a two-interval forced-choice procedure as described by Williams. ${ }^{12}$ The threshold was defined as $75 \%$-correct performance with 50 trials of a maximum-likelihood threshold-estimation procedure. $^{29}$ The stimuli were $500-\mathrm{ms}$ pulses separated by a $200-\mathrm{ms}$ interval. Retinal illuminance was $500 \mathrm{Td}$ at the center of the Gaussian luminance profile. On each trial, feedback was given to the observer to inform him or her whether a correct response had been made. In the third method, contrast sensitivity for static fringes was measured with the method of adjustment. We presented the stimulus for $500 \mathrm{~ms}$ every $2000 \mathrm{~ms}$ without changing the retinal illuminance. During testing with static gratings, the observer was allowed to control the relative phase of the fringes when necessary. When phase control was required by the observer, we set the contrast of the fringes temporarily at unity to make the adjustment easier.

Measurements were made at spatial frequencies of 2,4 , and $8 \mathrm{c} / \mathrm{deg}$. In a single session, contrast sensitivity for both isoluminant and isochromatic stimuli was measured with all three methods for one spatial frequency. In each session the observer made four settings with the method of adjustment and two runs of the forced-choice procedure. At least two sessions were run for all spatial frequencies. Two color-normal observers (OP and NS) were tested.

\section{Results}

Figure 5 shows the results for the two observers. For both isoluminant and isochromatic stimuli, the data from both observers show no statistically significant difference $(|p|>0.05$ except for isochromatic conditions of OP at $8 \mathrm{c} / \mathrm{deg}$, where $|p|>0.01$, t-test) between any two of three different measurements. This suggests that the drifting technique can provide an estimate of isoluminant and isochromatic contrast sensitivity. Furthermore, the fact that no systematic difference exists in the data obtained by two different experimental procedures for static gratings means that these two observers set their crite-
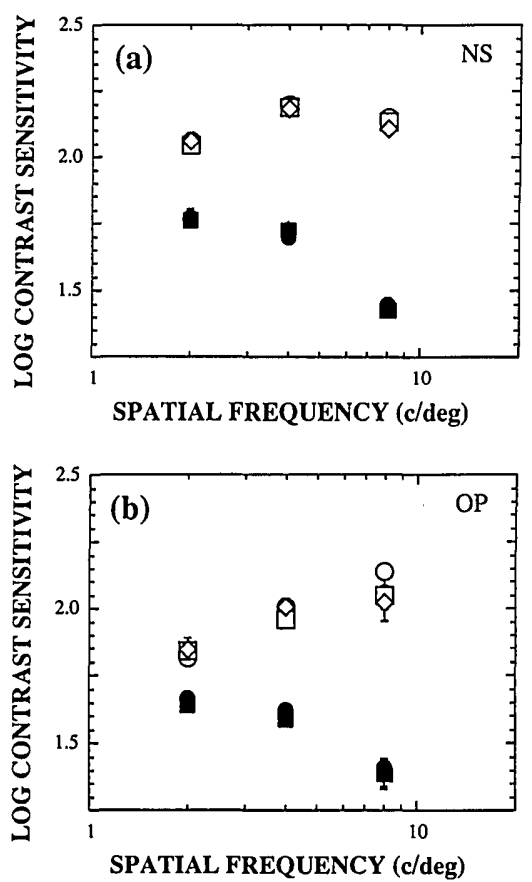

Fig. 5. Results of the validation experiment for observers (a) NS and (b) OP. In both panels, data are plotted in terms of the contrasts of both fringe components, which were equal. Filled symbols, data for isoluminant stimuli; open symbols, data for isochromatic stimuli. Circles, squares, and diamonds represent the data obtained with the drifting technique, with static fringes with the method of adjustment, and with static fringes with the two-alternative forced-choice method, respectively. Error bars show \pm 1 standard error of the mean. 
Table 1. Results of Foveal Resolution for Isoluminant and Isochromatic Interference Fringes

\begin{tabular}{cccccc}
\hline & \multicolumn{3}{c}{ Foveal Resolution Limit (c/deg) } \\
\cline { 2 - 3 } \cline { 5 - 5 } \cline { 5 - 6 } Observer & \multicolumn{2}{c}{ Isochromatic } & & \multicolumn{2}{c}{ Isoluminant } \\
\cline { 2 - 3 } \cline { 5 - 6 } Dean & Standard Error & & Mean & Standard Error \\
NS & 48.6 & 0.4 & & 27.0 & 0.1 \\
NS & 47.6 & 0.8 & & 22.5 & 0.1 \\
\hline
\end{tabular}

rion in the adjustment procedure close to that established by the forced-choice procedure. Head-movement artifacts forced us to limit this comparison to low spatial frequencies. We assume that the validation established at low frequencies holds at high frequencies, as well.

\section{EXPERIMENT 2}

\section{Method}

We measured the foveal resolution limit for both isoluminant and isochromatic modulations with the drifting technique. In order to produce continuous spatial-frequency adjustment, we replaced the final Maxwellian lens, L6 in Fig. 1, with a camera zoom lens (focal length $80-200 \mathrm{~mm}$ ). With this lens, the spatial-frequency range was set between 15 and $38 \mathrm{c} / \mathrm{deg}$ for isoluminant measurements and between 29 and $72 \mathrm{c} / \mathrm{deg}$ for isochromatic measurements. The red (632.8-nm) and the green (514.5-nm) interference fringes of unity contrast were windowed by a sharpedged circular aperture. No background was used. With these two fringes, the effective contrasts for the longwavelength-sensitive (L) and for the middle-wavelengthsensitive (M) cones produced by the isoluminant stimulus were $25 \%$ and $69 \%$, respectively. The size of the circular field decreases with increasing spatial frequency, with the number of grating cycles kept constant. In this experiment the diameter of the circular field was set at 6 deg for observer DW and at 2 deg for observer NS; these diameters were determined at 24 and $45 \mathrm{c} / \mathrm{deg}$ for isoluminant conditions and for isochromatic conditions, respectively. To measure the foveal resolution limit for isochromatic stimuli, the observer adjusted the power of the zoom lens so that the luminance modulation was just detectable. For isoluminant stimuli the observer adjusted the zoom lens so that the red-green modulation was just detectable. A total of eight settings, four each for isoluminant and for isochromatic modulations, were made by the observer. Two color-normal persons (DW and NS) served as observers.

\section{Results}

Results are shown in Table 1. For the isochromatic interference fringe, the foveal resolution limit for each observer is $\sim 48 \mathrm{c} / \mathrm{deg}$, slightly lower than the previous estimates of the foveal resolution limit. ${ }^{30,31}$ Since we did not use an incoherent background, this result is probably due to the influence of speckle masking. However, even though speckle noise lowers the visibility of the interference fringe, both observers saw red and green stripes at spatial frequencies higher than $20 \mathrm{c} / \mathrm{deg}$. The results show that the foveal resolution limits for observers DW and NS were 27 and $22.5 \mathrm{c} / \mathrm{deg}$, respectively. These are substantially higher than had previously been measured. An additional three color-normal observers (OP, DB, and SG) viewed the red and green interference fringes at $20 \mathrm{c} / \mathrm{deg}$ only, and all three reported resolving red and green stripes.

\section{DISCUSSION}

In this study a new psychophysical technique was developed for measuring isoluminant contrast sensitivity at middle and high spatial frequencies without the influence of diffraction and aberrations of the eye. With our technique we have clear evidence that the foveal resolution limit for the red and green isoluminant interference fringe exceeds $20 \mathrm{c} / \mathrm{deg}$.

\section{Appearance of the Test Stimulus}

The appearance of the test stimulus, the sum of a red and a green fringe of the same spatial properties drifting in opposite directions, can be described as a pair of counterphase gratings from which the isoluminant and the isochromatic modulation appear alternately. No particular motion (e.g., either right or left) can be perceived when the two fringes are approximately isoluminant. Our stimulus is similar to that used in the judging of isoluminance with a minimum-motion technique..$^{22,32,33}$ This stimulus consisted of two gratings: a red-and-green grating and a light-and-dark-yellow grating, each of which appeared alternately with the phase reversed from that of its predecessor. The apparent motion of the stimulus was minimized when the red and green bars were isoluminant. We observed the same phenomenon at isoluminance, although the motion null was not so clearly defined as the flicker nulls that we obtained with flicker photometry.

\section{Influence of Masking and Facilitation}

The results reveal additional important aspects of the use of the drifting technique. We found no evidence of masking or facilitation between the isoluminant and the isochromatic modulations; these effects have been reported for studies in which these two modulations are presented simultaneously. ${ }^{23,24}$ That such an interaction for the drifting stimulus was not observed is revealed by the good agreement of the contrast-sensitivity data obtained with the oppositely drifting fringes and the data obtained with the conventional static fringes (which contain either isoluminant or isochromatic modulation but not both). This result may be explained by the difference in temporal factors. The previous studies found masking or facilitation effects when isoluminant and isochromatic stimuli were presented simultaneously. On the other hand, our drifting fringes produce the isoluminant and the isochromatic fringes in temporal quadrature. The maximum fringe modulations appear alternately approximately every $1 \mathrm{~s}$. This temporal offset between isoluminant and isochromatic modulations probably reduces the interactions.

\section{Influence of Spatial-Frequency Adaptation}

Another important aspect to be considered is the influence of spatial-frequency adaptation. With oppositely drifting fringes, gratings of the same spatial frequency and orientation are presented more or less continuously, though the direction of modulation in color space is always changing. However, the good agreement between contrast sensitivities with the drifting and with the conventional techniques 
shows that spatial-frequency adaptation does not affect thresholds. This may be because the observer almost always saw low-contrast fringes during the experimental sessions; the contrast was low enough that significant adaptation effects were avoided.

\section{Foveval Resolution Limit at Isoluminance}

Our aberration-free measurements show that the foveal resolution limit for isoluminant gratings is $22.5-27 \mathrm{c} / \mathrm{deg}$. Three additional observers (DB, OP, and SG) also saw red and green fringes at least as high as $20 \mathrm{c} / \mathrm{deg}$. So far we have evidence for five observers. These are probably the first direct measurements that show that foveal resolution reaches such high spatial frequencies, and they agree well with those of Anderson et al., ${ }^{9}$ who reached a similar conclusion from extrapolation of data measured as high as $9 \mathrm{c} / \mathrm{deg}$. Cavonius and Schumacher ${ }^{34}$ reported a much higher foveal resolution limit (higher than $30 \mathrm{c} / \mathrm{deg}$ ) based on wavelength discrimination. However, they did not take steps to correct chromatic aberration, and thus there is no guarantee that their measurements were based only on isoluminant discriminations. The isoluminant resolution limit reported here places a lower bound on the sampling density of neurons that mediate color vision. For example, if the visual system reconstructs gratings only as high as the Nyquist frequency of the sampling array, then this array must have a sampling rate equal to twice the resolution limit. Caution must be exercised when one is applying this rule because of the possibility of superNyquist resolution. ${ }^{35}$

\section{Red-Green Fringes above the Resolution Limit}

Two color normals (DW and NS) who are experienced in seeing interference fringes observed isoluminant redgreen fringes of spatial frequency above its foveal resolution limit. Static red and green interference fringes of identical spatial properties were added out of phase at the fovea, and their contrast was set at $100 \%$. At $30 \mathrm{c} / \mathrm{deg}$, for example, the observers agreed that the fringe appeared to be filled with spatial noise of reddish and greenish colors. This noise is probably not caused by speckle noise in the apparatus, because when we magnify the fringe by putting a low-power lens in front of the eye, the noise disappears and red and green bars appear instead. Moreover, as the spatial frequency is reduced, the spatial noise can be seen outside the fovea, while a red-and-green grating can be resolved in the fovea. This dependence on spatial frequency is typical of aliasing phenomena observed with luminance gratings at much higher spatial frequencies. ${ }^{12}$

Other evidence supporting this view comes from observations made when we produce a beat pattern by slightly changing the spatial frequency or orientation of one of the fringes. The noise is observed in the beat pattern only where the two fringes are out of phase; no such spatial structure can be observed in the adjacent area where the fringes are in phase. This fact also supports the view that the noise is not generated by the eye's optics. The spatial noise generated by the eye's optics should have relatively constant properties across the retina and will not be localized at a particular location. So the noise is likely to be generated by some later stage in the visual system.

Williams et $a l .^{36}$ argued that the colors perceived from fine, periodic, achromatic patterns, originally described by
Brewster, ${ }^{37}$ can be explained by chromatic aliasing by the $\mathrm{L}$ and $\mathrm{M}$ cones. White and black gratings of $10-40 \mathrm{c} / \mathrm{deg}$ can appear to be covered with red and green splotches that are several times larger than the bars of the gratings. This color appearance can be produced by the local difference in the proportion of the $\mathrm{L}$ and $\mathrm{M}$ cones illuminated by bright bars. If the white and black gratings are replaced with red and green gratings of unresolvable spatial frequency, each cone or small group of cones produces its own output that depends on the wavelength, and this may produce spatially small color variations of a yellowish appearance. In this sense, the noise is similar to the phenomenon that tiny, brief yellow flashes presented at the fovea sometimes appear to be red or green, ${ }^{38-42}$ an effect that can be regarded as chromatic aliasing with a punctate stimulus. The noise observed from the isoluminant red and green interference fringe might be additional evidence of chromatic aliasing caused by the $\mathrm{L}$ and $\mathrm{M}$ cones or evidence for spatial sampling at a later stage in the chromatic pathway, but a complete explanation is not yet available.

Previous investigators $\mathrm{s}^{5,23}$ have suggested that isoluminant patterns of sufficiently high spatial frequency lose the appearance of color variation and look instead like luminance modulations. In contrast, our observers reported that when the chromatic resolution limit was exceeded, only the spatial noise was visible and there was no evidence that the isoluminant grating was perceived as a luminance grating. This observation makes it likely that the explanation for the earlier reports involves optical factors. For example, the effects of diffraction, axial chromatic aberration, or transverse chromatic aberration could produce a luminance artifact that is visible above the chromatic resolution limit.

\section{APPENDIX A: EFFECTS OF HEAD AND EYE MOVEMENTS}

In this appendix we estimate the size of the phase error between static red and green interference fringes produced by head and eye movements and the effect of this phase error on measured contrast sensitivity.

\section{Effect of Head and Eye Movements on the Relative Phase of Interference Fringes}

Suppose that the interfering beams enter the eye parallel to its optical axis and that the two pairs of interfering beams are symmetric about this optical axis. In this case there should be no optical path-length difference between the individual beams of either pair, and thus the two interference fringes would have the same spatial phase on the retina. (We assume, without loss of generality, that we have not adjusted the electromagnetic phase of either beam with the computer-controlled AOM's.) Head or eye movements change the optical path length for the interfering beams because they shift the optical elements of the eye relative to each pair of interfering beams. As a result of the shift, each beam is refracted differently at the optical interfaces. This asymmetry causes a shift in the spatial phase of the interference fringe that depends on its wavelength and spatial frequency. The amount of the phase shift depends on wavelengths, since for a fixed spatial frequency the beam separation at the corneal surface 

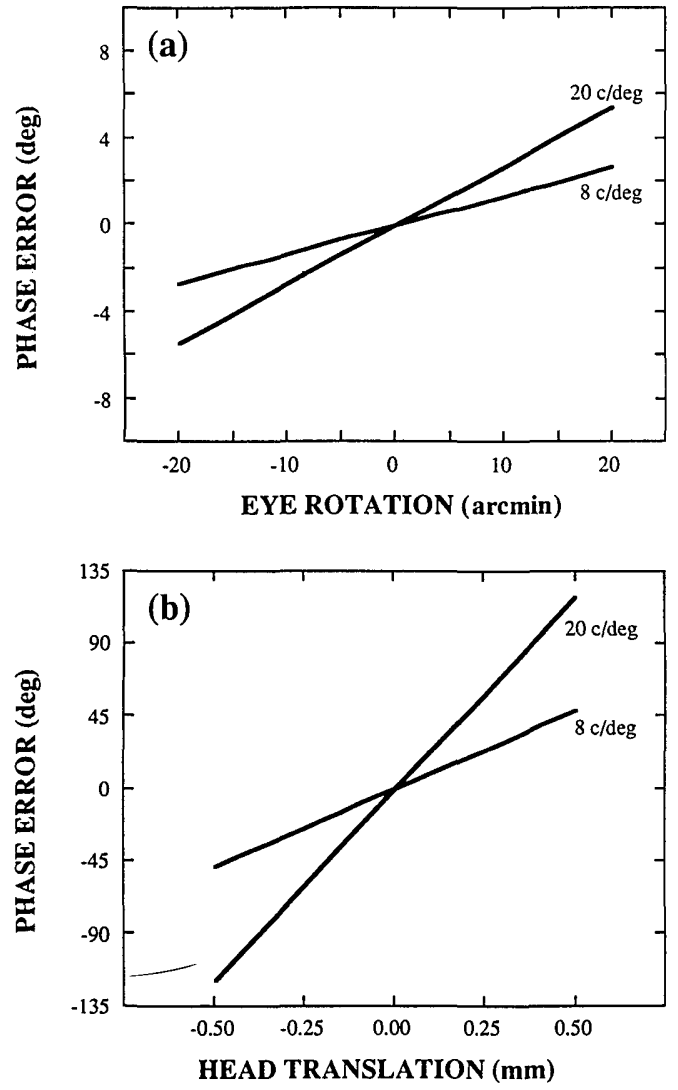

Fig. 6. Phase error caused by (a) eye rotation and (b) head translation between 632.8 - and 514.5-nm interference fringes.

is approximately proportional to wavelength, as shown by Eq. (1), and thus the effect is smaller for shorterwavelength components.

To estimate the relative phase shift that is due to the observer's head and eye movements for our red and green interference fringes, we made an optical ray tracing in which we used Snell's law to calculate the refraction at each optical interface. We used Le Grand's schematic eye model $^{43}$ to define the optical interfaces. This model provides a good description of axial chromatic aberration for the wavelengths that we used (514.5-632.8 nm). We modeled eye movements as rotations of the eye about its geometrical center and head movements as lateral shifts of the eye in a single plane. We traced the principal ray of each interfering beam and computed its optical path length as a function of head and eye position. The relative phase $\delta$ between the two interference fringes is given by

$$
\delta=\frac{\Delta \rho_{1}}{\lambda_{1}}-\frac{\Delta \rho_{2}}{\lambda_{2}}
$$

where $\lambda_{1}$ and $\lambda_{2}$ are the wavelengths of the two interference fringes and $\Delta \rho_{1}$ and $\Delta \rho_{2}$ are the optical path-length differences between the individual beams of each fringe. (The problem can be described in one spatial dimension because the two fringes have the same orientation: any movements of the eye or the head along the direction of the fringe bars produce no phase error.)

Figure 6 shows the estimates of the phase difference between 632.8 - and 514.5- $\mathrm{nm}$ interference fringes as a function of eye and head movements. The calculations were made for the fringe spatial frequencies of 8 and
$20 \mathrm{c} / \mathrm{deg}$. Figure 6(a) shows the phase error as a function of eye rotation, assuming that the observer tries to fixate the test stimulus. The phase error increases monotonically with the amount of eye rotation and the fringe spatial frequency. The size of fixational eye movements is estimated to be as large as 10 arcmin. ${ }^{4-47}$ The phase error between 632.8- and 514.5-nm interference fringes produced by an eye movement of $10 \mathrm{arcmin}$ is $\sim 1.5 \mathrm{deg}$ at $8 \mathrm{c} / \mathrm{deg}$ and $2.7 \mathrm{deg}$ at $20 \mathrm{c} / \mathrm{deg}$. Figure 6(b) shows the phase error as a function of head translation. The results show that the phase error increases monotonically with the amount of head translation and the fringe spatial frequency. Since we are unaware of direct measurements of the size of head translations for an observer clenching a bite bar, we made estimates based on the conservative assumption that all the within-session variability in measurements of the Stiles-Crawford maximum made by Williams ${ }^{48}$ and those of transverse chromatic aberration made by Simonet and Campbell ${ }^{49}$ was caused by head movements. Our calculation based on that assumption suggests that typical head translations range between 130 and $460 \mu \mathrm{m}$. A lateral head movement of $300 \mu \mathrm{m}$, for example, produces a phase error of $30 \mathrm{deg}$ at $8 \mathrm{c} / \mathrm{deg}$ and $70 \mathrm{deg}$ at $20 \mathrm{c} / \mathrm{deg}$, each of which is more than 20 times greater than that from typical fixational eye movements.

Effect of Phase Error on Measured Contrast Sensitivity To understand the effect of head and eye movements on red-green-isoluminant contrast sensitivity measured with static interference fringes, we estimated the effect of the phase error between the two components on measured contrast sensitivity. To do this, we required a model for how the visual system detects sinusoidal gratings. In the calculations that follow, we assume that the detection of our red and green interference fringes is mediated by the responses $R_{L}$ and $R_{C}$ of a luminance mechanism and a chromatic mechanism, respectively. Each mechanism responds linearly to grating contrast, but the two mechanisms are characterized by different spectral responsivity functions: the luminance mechanism does not respond to spatial modulations that are isoluminant, and the chromatic mechanism does not respond to spatial modulation that have a constant chromaticity. We assume a vector length model of summation between the responses of the two mechanisms ${ }^{50-52}$ so that a stimulus is above contrast threshold when

$$
R=\left(R_{L}^{2}+R_{C}^{2}\right)^{1 / 2} \geq 1
$$

For any grating spatial frequency, we let the constants $T_{L}$ and $T_{C}$ represent contrast threshold for gratings that stimulate each mechanism alone. We can write the mechanism responses in terms of the contrasts $C_{L}$ and $C_{C}$ seen by each mechanism and of these contrast thresholds: $R_{L}=C_{L} / T_{L}$ and $R_{C}=C_{C} / T_{C}$. We allow the contrast thresholds for each mechanism to depend on grating spatial frequency, but we assume that the spectral responsivity of each mechanism is independent of this spatial frequency. ${ }^{53,54}$ We also assume that the response of each mechanism does not depend on the spatial phase of the grating.

We next determine $C_{L}$ and $C_{C}$ as a function of the phase error between the two fringes. Recall that static red- 
green-isoluminant fringe stimuli consist of a red (632.8$\mathrm{nm})$ and a green (514.5- $\mathrm{nm}$ ) interference fringe of the same spatial frequency, $f$, orientation, and contrast, $C$, which would be in spatial counterphase in the absence of eye and head movements. Since the incoherent background is composed of the same wavelengths as those of the interference fringes, and since the retinal illuminances of the two background components are in the same ratio as that of the interference fringes, the effect of the background can be incorporated directly into the common contrast, $C$. We ignore effects of the Gaussian window. The intensity profiles of the red and the green components of the stimulus, $I_{R}$ and $I_{G}$, respectively, can be described as

$$
\begin{aligned}
& I_{R}(x)=E_{R}\left[1+C \sin \left(f x+\frac{\delta}{2}\right)\right], \\
& I_{G}(x)=E_{G}\left[1-C \sin \left(f x-\frac{\delta}{2}\right)\right],
\end{aligned}
$$

where $E_{R}$ and $E_{G}$ represent the mean radiance of the red and the green interference fringes, respectively, and $\delta$ represents the phase error between them $(\delta=0$ indicates spatial counterphase). When the fringes are superimposed upon the retina, the spatial modulation seen by the luminance mechanism is given by

$$
I_{L}(x)=I_{R}(x) S_{L}\left(\lambda_{R}\right)+I_{G}(x) S_{L}\left(\lambda_{G}\right),
$$

where $\lambda_{R}$ is the wavelength of the red interference fringe, $\lambda_{G}$ is the wavelength of the green interference fringe, and $S_{L}(\lambda)$ is the spectral responsivity of the luminance mechanism. By substituting for $I_{R}(x)$ and $I_{G}(x)$ and then simplifying with the use of standard trigonometric identities, we can rewrite the modulation seen by the luminance mechanism as

$$
\begin{aligned}
I_{L}(x)= & K_{L}^{\delta=\pi}+C\left\{\left[K_{L}^{\delta=0} \cos (\delta / 2)\right]^{2}\right. \\
& \left.+\left[K_{L}^{\delta=\pi} \sin (\delta / 2)\right]^{2}\right\}^{1 / 2} \sin \left[f x-\varphi_{L}(\delta)\right],
\end{aligned}
$$

where $K_{L}^{\delta=0}=E_{R} S_{L}\left(\lambda_{R}\right)-E_{G} S_{L}\left(\lambda_{G}\right)$, and $K_{L}^{\delta=\pi}=$ $E_{R} S_{L}\left(\lambda_{R}\right)+E_{G} S_{L}\left(\lambda_{G}\right)$. The exact form of the phase $\varphi_{L}(\delta)$ is not critical for the calculations that follow. Equation (A5) tells us that as the phase error $\delta$ between the two interference fringes changes, the modulation seen by the luminance mechanism is always a sinusoid with mean level $K_{L}^{\delta=\pi}$. Both the amplitude and the phase of this sinusoid vary with $\delta$. The constants $\left|K_{L}^{\delta=0}\right|$ and $\left|K_{L}^{\delta=\pi}\right|$ specify, for any contrast $C$, the amplitude of the sinusoid seen by the luminance mechanism when the two fringes are in spatial counterphase and in spatial register, respectively. Similarly, we can describe the modulation seen by the chromatic mechanism as

$$
\begin{aligned}
I_{C}(x)= & K_{C}^{\delta=\pi}+C\left\{\left[K_{C}{ }^{\delta=0} \cos (\delta / 2)\right]^{2}\right. \\
& \left.+\left[K_{C}^{\delta=\pi} \sin (\delta / 2)\right]^{2}\right\}^{1 / 2} \sin \left[f x-\varphi_{C}(\delta)\right],
\end{aligned}
$$

where the definitions of $K_{C}{ }^{\delta=0}, K_{C}{ }^{\delta=\pi}$, and $\varphi_{C}(\delta)$ are analogous to those of $K_{L}^{\delta=0}, K_{L}^{\delta=\pi}$, and $\varphi_{L}(\delta)$, with the exception that the spectral responsivity function of the luminance mechanism $S_{L}(\lambda)$ is replaced by the spectral responsivity function of the chromatic mechanism $S_{C}(\lambda)$. Because our red and green interference fringes are isoluminant, we know that the luminance mechanism does not respond when the fringes are presented in spatial counterphase so that $K_{L}{ }^{\delta=0}=0$. Because the chromatic mechanism does not respond to modulations of constant chromaticity, we also have $K_{C}{ }^{\delta=\pi}=0$. Thus the amplitudes of the modulations seen by the luminance and the chromatic mechanisms as a function of the phase error between the red and the green fringes are given by the expressions $C\left|K_{L}{ }^{\delta=\pi}\right| \sin (\delta / 2)$ and $C\left|K_{C}{ }^{\delta=0}\right| \cos (\delta / 2)$, respectively. We define contrast scales for the luminance and the chromatic mechanisms so that mechanism contrast corresponds to the common fringe contrast $C$ when the fringes are perfectly in phase $(\delta=\pi)$ (for the luminance mechanism) and perfectly out of phase $(\delta=0)$ (for the chromatic mechanism). From the preceding expressions for amplitude we see that this implies that the contrast seen by the luminance mechanism as a function of phase error is given by $C_{L}(\delta)=C \sin (\delta / 2)$, while contrast seen by the chromatic mechanism is given by $C_{C}(\delta)=C \cos (\delta / 2)$. Combining these two expressions with relation (A2), incorporating the relation between contrast and mechanism response, and solving for contrast threshold, we have that measured contrast threshold depends on the phase error according to

$$
C(\delta)=1 /\left\{\left[\frac{\sin (\delta / 2)}{T_{L}}\right]^{2}+\left[\frac{\cos (\delta / 2)}{T_{C}}\right]^{2}\right\}^{1 / 2} .
$$

We define contrast sensitivity as the reciprocal of contrast threshold and let $k$ represent the ratio of measured contrast sensitivity for isochromatic stimuli to that for isoluminant stimuli: $k=T_{C} / T_{L}$. Then we can compute the ratio of the measured contrast sensitivity $[1 / C(\delta)]$ to the contrast sensitivity that we would like to measure $\left(1 / T_{C}\right)$ as a function of phase error by

$$
T_{C} / C(\delta)=\left\{[k \sin (\delta / 2)]^{2}+[\cos (\delta / 2)]^{2}\right\}^{1 / 2} .
$$

Figure 7(a) plots the effect of the phase error on measured isoluminant contrast sensitivity for two different choices of $k$. Preliminary experiments show that the ratio of contrast sensitivity for isochromatic stimuli to that for the isoluminant stimuli is $\sim 0.5 \log$ unit at $8 \mathrm{c} / \mathrm{deg}$ and $\sim 1.0 \log$ unit at $20 \mathrm{c} / \mathrm{deg}$. For these choices of $k$, our calculations show that measured isoluminant contrast sensitivity is lowest when there is no phase error between the two grating components. That is, a phase error between the two fringes would make isoluminant contrast sensitivity appear to be higher than it really is. At $20 \mathrm{c} / \mathrm{deg}$ (which roughly corresponds to the curve labeled $1.0 \mathrm{LOG}$ ), our calculations suggest that the phase error must be less than $8.8 \mathrm{deg}$ for measurement of the isoluminant contrast sensitivity with an error smaller than 0.1 log unit. At $8 \mathrm{c} / \mathrm{deg}$ (which roughly corresponds to the curve labeled $0.5 \mathrm{LOG})$, the corresponding phase error is $29.5 \mathrm{deg}$.

In Fig. 7(b) the predicted error in contrast-sensitivity measurements for isoluminant fringes is shown replotted as a function of head translation. For both 8 and $20 \mathrm{c} / \mathrm{deg}$, head translation increases the estimate of isoluminant contrast sensitivity. The effect is more severe at the higher spatial frequency. Our calculations suggest that for measuring isoluminant contrast sensitivity with an error smaller than $0.1 \mathrm{log}$ unit at $20 \mathrm{c} / \mathrm{deg}$, the tolerable 

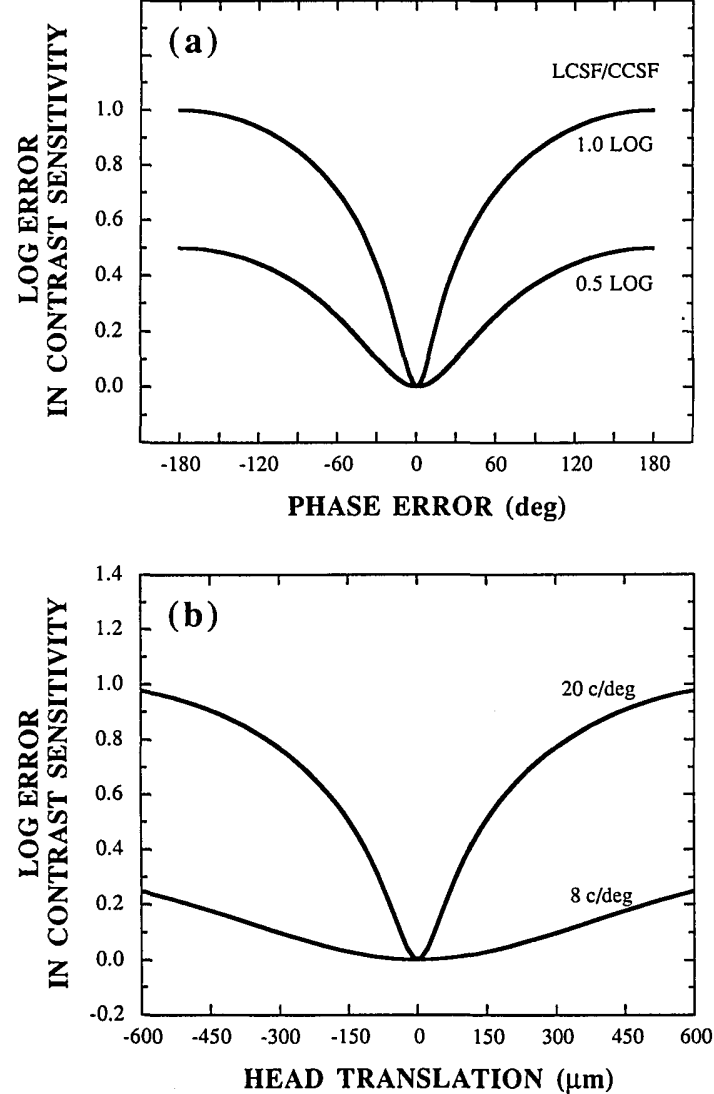

Fig. 7. (a) Effect of phase error on isoluminant contrast sensitivity, (b) effect of head translation on isoluminant contrast sensitivity.

head translation can be no more than $30 \mu \mathrm{m}$, which may be difficult to achieve. At $8 \mathrm{c} / \mathrm{deg}$, however, the tolerable head translation can be as high as $304 \mu \mathrm{m}$. This is comparable with the size of typical head movements that we estimated above. This suggests that $8 \mathrm{c} / \mathrm{deg}$ is approximately the highest spatial frequency at which we can accurately measure isoluminant contrast sensitivity with static red-green interference fringes. The phase errors caused by eye movements are so small that they produce negligible distortion in measured contrast sensitivity, even at high spatial frequencies. We have also performed our calculations with a model of contrast sensitivity that assumes that the two mechanisms contribute independently to grating detection. ${ }^{53,54}$ The results of these calculations lead us to substantially the same conclusions about the acceptable phase error.

\section{APPENDIX B: EFFECTS OF ISOLUMINANCE ERROR}

This appendix provides an estimate of the difference in the luminance of the red and the green fringes on measured isoluminant contrast sensitivity. Such a difference will produce a luminance artifact, so that detection is mediated by both the luminance and the chromatic mechanisms. We make the computation for the case in which the gratings are presented in exact counterphase, corresponding to $\delta=0$, as defined in Appendix A. Starting with Eq. (A5), substituting for $\delta=0$, and using the same definition of contrast as in Appendix A, we can derive an expression for the contrast seen by the luminance mechanism as a function of the radiances $E_{R}$ and $E_{G}$ of the red and the green fringes, respectively. This expression is

$$
C_{L}\left(E_{R}, E_{G}\right)=\frac{C\left|K_{L}^{\delta=0}\right|}{\left|K_{L}^{\delta=\pi}\right|}=\frac{C\left|E_{R} S_{L}\left(\lambda_{R}\right)-E_{G} S_{L}\left(\lambda_{G}\right)\right|}{\left|E_{R} S_{L}\left(\lambda_{R}\right)+E_{G} S_{L}\left(\lambda_{G}\right)\right|} .
$$

Similarly, we can start with Eq. (A6) and derive for the chromatic mechanism that

$$
C_{C}\left(E_{R}, E_{G}\right)=C \text {. }
$$

The contrast seen by the chromatic mechanism does not change with $E_{R}$ and $E_{G}$, because this mechanism is assumed to adapt so that it never sees modulations of constant chromaticity.

If we let $K$ represent the ratio of the luminance of the red component to that of the green component, $K=$ $E_{R} S_{L}\left(\lambda_{R}\right) / E_{G} S_{L}\left(\lambda_{G}\right)$, then we can simplify Eq. (B1) to $C_{L}(K)=C|1-K| /|1+K|$. From this we can compute the ratio of measured contrast sensitivity to red-greenisoluminant contrast sensitivity by

$$
T_{C} / C(K)=\left\{[k(1-K) /(1+K)]^{2}+1\right\}^{1 / 2},
$$

where $k$ is again the ratio of isochromatic to isoluminant contrast sensitivity.

Figure 8 shows the effects of an error in the luminance ratio on isoluminant contrast sensitivity. The artifact produced by an error in the luminance ratio is typically much less severe than that produced by a phase error. For example, if the ratio of contrast sensitivity for the isochromatic stimuli to that for the isoluminant stimuli is $1.0 \mathrm{log}$ unit, which it is at roughly $20 \mathrm{c} / \mathrm{deg}$, our calculations suggest that the tolerable error in the luminance ratio is within the range $\pm 16.5 \%$ for measuring red-greenisoluminant contrast sensitivity data with an accuracy of $0.1 \mathrm{log}$ unit. The standard errors in flicker photometry are typically very small. In our case the $99 \%$ confidence interval for the mean setting is less than $\pm 10 \%$. Thus we conclude that errors in the luminance ratio of our red to green fringes are unlikely to have contaminated our measurements of isoluminant contrast sensitivity. We have also performed our calculations with a model of contrast sensitivity that assumes that the two mechanisms contribute independently to grating detection. ${ }^{53,54}$ The results of these calculations lead us to similar conclusions about the effect of luminance error on our measurements.

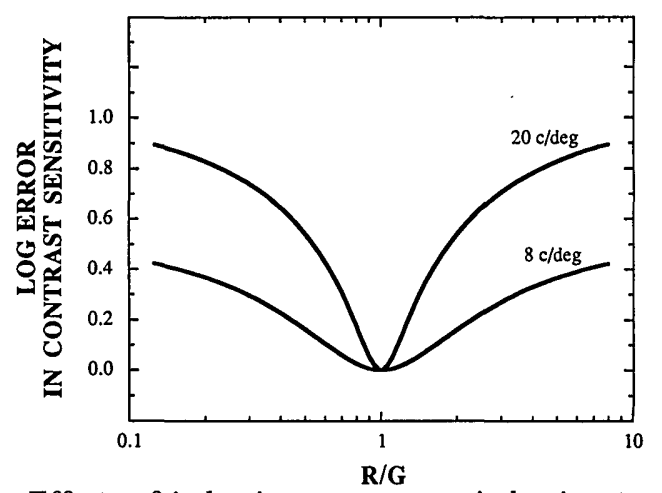

Fig. 8. Effects of isoluminance error on isoluminant contrast sensitivity. 


\section{ACKNOWLEDGMENTS}

We thank Arthur Bradley for his comments on an earlier version of this manuscript. We also thank Orin Packer and Sue Galvin for serving as observers and Bill Haake and Alan Russell for technical assistance. This research was supported by grants EY04367, EY01319, EY06278, and EY09625 from the National Institutes of Health.

*Present address, Basic Research Department, Olympus Optical Co., Ltd., 2-3 Kuboyama, Hachioji, Tokyo 192 Japan.

${ }^{\dagger}$ Present address, Department of Psychology, University of California, Santa Barbara, Santa Barbara, California 93106

\section{REFERENCES}

1. O. H. Schade, "On the quality of color-television images and the perception of color detail," J. Soc. Motion Picture Telev. Eng. 67, 801-819 (1958).

2. G. J. C. Van der Horst, C. M. M. De Weert, and M. A. Boumann, "Transfer of spatial chromaticity-contrast at threshold in the human eye," J. Opt. Soc. Am. 57, 1260-1266 (1967).

3. G. J. C. Van der Horst and M. A. Boumann, "Spatiotemporal chromaticity discrimination," J. Opt. Soc. Am. 59, 14821488 (1969).

4. R. Hilz and C. R. Cavonius, "Wavelength discrimination measured with square-wave gratings," J. Opt. Soc. Am. 60, 273277 (1970).

5. E. M. Granger and J. C. Heurtley, "Visual chromaticitymodulation transfer function," J. Opt. Soc. Am. 63, 11731174 (1973).

6. C. Noorlander, M. J. G. Heuts, and J. J. Koenderink, "Influence of the target size on the detection threshold for luminance and chromaticity contrast," J. Opt. Soc. Am. 70, 1116-1121 (1980).

7. K. T. Mullen, "The contrast sensitivity of human color vision to red-green and blue-yellow chromatic gratings," J. Physiol. 359, 381-400 (1985).

8. K. T. Mullen, "Colour vision as a post-receptoral specialization of the central visual field," Vision Res. 31, 119-130 (1991).

9. S. J. Anderson, K. T. Mullen, and R. F. Hess, "Human peripheral spatial resolution for achromatic and chromatic stimuli: limits imposed by optical and retinal factors," J. Physiol. 442, 47-64 (1991).

10. Y. Le Grand, "Sur la mesure de l'acuité visuelle au moyen de franges d'interférence," C. R. Acad. Sci. Paris 200, 490-491.

11. F. W. Campbell and D. G. Green, "Optical and retinal factors affecting visual resolution," J. Physiol. 181, 576-593 (1965).

12. D. R. Williams, "Aliasing in human foveal vision," Vision Res. 25, 195-205 (1985).

13. L. N. Thibos, A. Bradley, and D. L. Still, "Interferometric measurement of visual acuity and the effect of ocular chromatic aberration," Appl. Opt. 30, 2079-2087 (1991).

14. N. Sekiguchi, D. R. Williams, and D. H. Brainard, "Efficiency in detection of isoluminant and isochromatic interference fringes," J. Opt. Soc. Am. A 10, 2118-2133 (1993).

15. S. Mallick, "Common-path interferometers," in Optical Shop Testing, D. Malacara, ed. (Wiley, New York, 1978), pp. 81104.

16. W. H. Steel, Interferometry, 2nd ed. (Cambridge U. Press, Cambridge, 1983).

17. D. R. Williams, "Visibility of interference fringes near the resolution limit," J. Opt. Soc. Am. A 2, 1087-1093 (1985).

18. N. J. Coletta, D. R. Williams, and C. L. M. Tiana, "Consequences of spatial sampling for human motion perception," Vision Res. 30, 1631-1648 (1990).

19. G. J. Burton, "Evidence for non-linear response processes in the human visual system from measurements on the threshold of spatial beat frequencies," Vision Res. 13, 1211-1225 (1973).
20. D. I. A. MacLeod, D. R. Williams, and W. Makous, "A visual nonlinearity fed by single cones," Vision Res. 32, 347-363 (1992).

21. N. Sekiguchi, D. R. Williams, and O. Packer, "Nonlinear distortion of gratings at the foveal resolution limit," Vision Res. 31, 815-831 (1991).

22. S. Anstis and P. Cavanagh, "A minimum motion technique for judging equiluminance," in Colour Vision, J. D. Mollon and L. T. Sharpe, eds. (Academic, London, 1983), pp. 155-166.

23. K. K. De Valois and E. Switkes, "Simultaneous masking interactions between chromatic and luminance gratings," J. Opt. Soc. Am. 73, 11-18 (1983).

24. E. Switkes, A. Bradley, and K. K. De Valois, "Contrast dependence and mechanisms of masking interactions among chromatic and luminance gratings," J. Opt. Soc. Am. A 5, 1149-1162 (1988).

25. F. W. Campbell and R. W. Gubisch, "The effect of chromatic aberration on visual acuity," J. Physiol. 192, 345-358 (1966).

26. X. Zhang, A. Bradley, and L. N. Thibos, "Achromatizing the human eye: the problem of chromatic parallax," J. Opt. Soc. Am. A 8, 686-691 (1991).

27. F. W. Campbell, R. H. S. Carpenter, and J. Z. Levinson, "Visibility of aperiodic patterns compared with that of sinusoidal gratings," J. Physiol. 204, 283-298 (1969).

28. D. H. Kelly, "Effects of sharp edges on the visibility of sinusoidal gratings," J. Opt. Soc. Am. 60, 98-103 (1970).

29. A. B. Watson and D. G. Pelli, "QUEST: a Bayesian adaptive psychometric method," Percept. Psychophys. 33, 113-120 (1983).

30. O. Packer, D. R. Williams, N. Sekiguchi, N. J. Coletta, and S. Galvin, "Effects of chromatic adaptation on foveal acuity and aliasing," Invest. Ophthal. Vis. Sci. Suppl. 30, 53 (1989).

31. O. Packer and D. R. Williams, "Blurring by fixational eye movements," Vision Res. 32, 1931-1939 (1992).

32. P. Cavanagh, S. Anstis, and G. Mather, "Screening for color blindness using optokinetic nystagmus," Invest. Ophthalmol. Vis. Sci. 25, 463-466 (1984).

33. P. Cavanagh, D. I. A. MacLeod, and S. M. Anstis, "Equiluminance: spatial and temporal factors and the contribution of blue-sensitive cones," J. Opt. Soc. Am. A 4, 1428-1438 (1987).

34. C. R. Cavonius and A. W. Schumacher, "Human visual acuity measured with colored test objects," Science 152, 1276-1277 (1966).

35. D. R. Williams and N. J. Coletta, "Cone spacing and the visual resolution limit," J. Opt. Soc. Am. A 4, 1514-1523 (1987).

36. D. R. Williams, N. Sekiguchi, W. Haake, D. Brainard, and O. Packer, "The cost of trichromacy for spatial vision," in From Pigments to Perception, A. Valberg and B. B. Lee, eds. (Plenum, New York, 1991), pp. 11-22.

37. D. Brewster, "On the undulations excited in the retina by the action of luminous points and lines," London Edinburgh Philos. Mag. J. Sci. 1, 169-174 (1832).

38. J. Krauskopf, "Color appearance of small stimuli and the spatial distribution of color receptors," J. Opt. Soc. Am. 54, 1171 (1964).

39. J. Krauskopf, "On identifying detectors," in Visual Psychophysics and Physiology, J. C. Armington, J. Krauskopf, and B. R. Wooten, eds. (Academic, New York, 1978), pp. 283-295.

40. J. Krauskopf and R. Srebro, "Spectral sensitivity of color mechanisms: derivation from fluctuations of color appearance near threshold," Science 150, 1477-1479 (1965).

41. C. M. Cicerone and J. L. Nerger, "The relative numbers of long-wavelength-sensitive to middle-wavelength-sensitive cones in the human fovea centralis," Vision Res. 29, 115-128 (1989).

42. R. L. P. Vimal, J. Pokorny, V. C. Smith, and S. K. Shevell, "Foveal cone thresholds," Vision Res. 29, 61-78 (1989).

43. Y. Le Grand, Form and Space Vision, M. Milldot and G. G. Heath, eds. (Indiana U. Press, Bloomington, Ind., 1967), pp. 5-23.

44. L. Riggs and J. C. Armington, "Motions of the retinal image during fixation," J. Opt. Soc. Am. 44, 315-321 (1954).

45. H. C. Bennet-Clark, "I'he oculomotor response to small target displacements," Opt. Acta 11, 301-314 (1964).

46. R. M. Steinman, G. M. Haddad, A. A. Skavenski, and D. Wyman, "Miniature eye movement," Science 181, 810-819 (1973). 
47. M. Eizenman, P. E. Hallett, and R. C. Frecker, "Power spectra for ocular drift and tremor," Vision Res. 25, 1635-1640 (1985).

48. D. R. Williams, "Visual consequences of the foveal pit," Invest. Ophthalmol. Vis. Sci. 19, 653-667 (1980).

49. P. Simonet and M. C. W. Campbell, "The optical transverse chromatic aberration on the fovea of the human eye," Vision Res. 31, 187-206 (1990).

50. A. B. Poirson and B. A. Wandell, "Task-dependent color discrimination," J. Opt. Soc. Am. A 7, 776-782 (1990).

51. A. B. Poirson, B. A. Wandell, D. C. Verner, and D. H. Brainard,
"Surface characterizations of color thresholds," J. Opt. Soc. Am. A 7, 783-789 (1990).

52. A. B. Poirson, "Appearance and detection of colored patterns," Ph.D. dissertation (Stanford University, Stanford, Calif., 1992).

53. K. Kranda and P. E. King-Smith, "Detection of coloured stimuli by independent linear systems," Vision Res. 19, 733746 (1979).

54. C. F. Stromeyer III, G. R. Cole, and R. E. Kronauer, "Secondsite adaptation in the red-green chromatic pathways," Vision Res. 25, 219-237 (1985). 